\title{
Longitudinal blood cell transcriptomic profiling and in vitro temporal proteomics provides novel insights into metabolic reprogramming and host-immune responses against Crimean-Congo Hemorrhagic Fever Virus
}

Ujjwal Neogi ( $\nabla$ ujjwal.neogi@ki.se )

Karolinska Institutet https://orcid.org/0000-0002-0844-3338

Nazif Elaldi

Sivas Cumhuriyet University

Sofia Appelberg

Public Health Agency of Sweden

Anoop Ambikan

Karolinska Institute

Emma Kennedy

Public Health England

Stuart Dowall

Public Health England, Porton Down,

Binnur Bagci

Sivas Cumhuriyet University

Soham Gupta

Karolinska Institutet

Jimmy Rodriguez

Karolinska Institutet https://orcid.org/0000-0002-6735-3332

Sara Svensson Akusjärvi

Karolinska Institute

Vanessa Monteil

Karolinska Institutet https://orcid.org/0000-0002-2652-5695

Akos Vegvari

Karolinska Institutet https://orcid.org/0000-0002-1287-0906

Akhil Banerjea

Institute of Advanced Virology

Friedemann Weber 
Institute for Virology, FB10-Veterinary Medicine, Justus-Liebig University Giessen https://orcid.org/0000-0001-9737-337X

\section{Roger Hewson}

Public Health England, Porton Down, https://orcid.org/0000-0003-2273-3152

\section{Ali Mirazimi ( $\nabla$ ali.mirazimi@ki.se )}

Department of Laboratory Medicine (LABMED), Karolinska Institute https://orcid.org/0000-0003-23716055

\section{Article}

Keywords: Crimean-Congo hemorrhagic fever orthonairovirus (CCHFV), pathogenesis, host immune response, peripheral blood mononuclear cells, longitudinal cohort

Posted Date: May 6th, 2021

DOl: https://doi.org/10.21203/rs.3.rs-465746/v1

License: (c) (1) This work is licensed under a Creative Commons Attribution 4.0 International License. Read Full License 


\section{Abstract}

The pathogenesis and host-viral interactions of the Crimean-Congo hemorrhagic fever orthonairovirus (CCHFV) are convoluted and is not well evaluated. To understand the host immune responses against CCHFV, we have performed a global transcriptomic analysis of peripheral blood mononuclear cells from a longitudinal cohort of CCHF patients who survived the infection and temporal untargeted proteomics analysis of CCHFV infected Huh7 cells. Our results indicate that during the acute phase of CCHFV infection, the host's metabolic reprogramming towards central carbon metabolism including glycolysis and glutaminolysis occurs that favours the virus replication as blocking these pathways in vitro inhibits CCHFV cellular replication. Furthermore, CCHFV replication was inhibited by blocking Akt with MK-2206 suggesting a regulatory role of PI3K/Akt/mTOR pathways. We also show activation of key interferon stimulating genes during infection, suggesting a role for type I and II interferon-mediated antiviral mechanisms. Targeting immune-metabolic pathways could be attractive therapeutic intervention for CCHFV.

\section{Introduction}

Crimean-Congo hemorrhagic fever orthonairovirus (CCHFV) is a negative-sense RNA virus belonging to the Nairoviridae virus family, which causes a mild to severe viral hemorrhagic fever and is a major emerging viral threat causing an increasing number of outbreaks in several parts of the globe. CrimeanCongo hemorrhagic fever (CCHF) poses a substantial threat to public health due to its high mortality rate in humans (3-40\%), modes of transmission (tick-to-human/animal, animal-to-human and human-tohuman) and geographical distribution ${ }^{1}$. CCHF is endemic in almost 30 countries in sub-Saharan Africa, South-Eastern Europe, the Middle East, Central Asia ${ }^{2,3}$. The Ixodid ticks, especially those of the genus, Hyalomma, are both a vector and a reservoir for CCHFV and are highly ubiquitous with its presence in more than 40 countries $^{4}$. In recent years, CCHFV outbreaks have become more frequent and have spread to new geographical areas. This has been attributed to climate changes and the spread of infected ticks by birds and the livestock trade. The presence of the CCHFV vector in Portugal, Spain, Germany, and even Sweden ${ }^{5}$ and England ${ }^{6}$ highlights the need for stricter surveillance due to the possibility of a future epidemic ${ }^{7}$.

Viruses exploit the host metabolic machinery to meet their biosynthetic demands. This reliance is further highlighted by observed variations in the cell-specific viral replications and production leading to changes in host metabolism ${ }^{8}$. In particular, virus infection leads to significant metabolic alterations in the host, in some cases resulting in changes in glycolysis rates and ATP production rates ${ }^{9}$. The changes in the energy metabolism can therefore be seen as an evolving property of the combined host-virus metabolic system and could be related to changes in host cellular demands arising from viral production ${ }^{10}$.

One of the primary antiviral defense mechanisms is type-I interferon (IFN-I) response. IFN-I are pleiotropic cytokines with varied cellular functions mediated by the transcriptional activations of several interferon- 
stimulated genes (ISGs). It is known that CCHFV replication is sensitive to IFN- $\mathrm{I}^{11}$. However, the virus is also able to delay the induction of IFN, and IFN treatment has been shown to be ineffective following establishment of infection, suggesting that CCHFV has developed mechanisms to block innate immune responses ${ }^{12}$. The existing knowledge from the in vitro studies, autopsies of CCHF patients and clinical findings suggests a regulatory mechanism of the IFN-response during CCHFV infection and indicates that ISGs may offer protection by inhibiting CCHFV. Furthermore, It has been suggested that many of the ISGs can rewire cellular metabolism to support an antiviral response. ${ }^{13}$ However, not much is known about the induction of ISGs during CCHFV infection and their relationship with metabolic pathways.

Because of the sporadic nature of CCHF outbreaks in humans in the endemic regions, a lack of infrastructure, and the absence of structured studies, little is known about the pathogenesis and hostvirus interactions during the acute phase of CCHF disease and associated sequelae after recovery. Understanding the host response to CCHFV is necessary to design better therapeutic and containment strategies for CCHF. Recently, systems biology studies using -omics approaches on patient material and infected cells have offered a route to elucidate potential mechanisms of the host immune response against invading viruses and disease pathogenesis, as recently reported by us in case of SARS-CoV-2 infection $^{14}$.

In this study, we have applied global blood transcriptomics in longitudinal blood samples collected during both the acute phase of CCHFV infection and the convalescent-phase (nearly after a year of recovery) and temporal quantitative proteomics analysis and modulation of the key pathways by drugs in in vitro infection model to understand the host immune and cellular responses against CCHFV. Our study provides a comprehensive understanding of the regulation of cellular pathways during CCHFV infection.

\section{Results}

Samples and clinical data. In this study, 18 samples were collected during the acute phase of the disease with a median time of 4 days (range 1-6 days) after the onset of symptoms. By using severity grading scores (SGS), 33\% (6/18) patients were grouped into severity group 1 (SG-1), 61\% (11/18) patients into severity group 2 (SG-2) and, $6 \%$ (1/18) patients into severity group 3 (SG-3). The median age of the patients was 49 years (range: 18-79), and $12(66.7 \%)$ of the patients were male. A 79-year-old male patient in SG-3 died on the third day of hospitalization. The case-fatality-rate (CFR) for the cohort was $5.6 \%$. Follow-up samples were collected from 12 individuals after a median duration of 54 weeks (range: 46-57 weeks). The CCHF patient characteristics are summarized individually in Supplementary Table S1.

\section{The impact of CCHF severity on peripheral blood transcriptome and cytokine profile during acute}

infection. We first considered the effect of the SGS disease severity group on the gene expression profile at the mRNA level during the acute phase of infection. The UMAP using all of the genes (Fig. S1A) and hierarchical clustering analysis of the top 500 high variable genes (Fig. S1B) did not show distinct differences between samples of different severity groups. To specifically investigate the genes that were significantly associated with disease severity, the samples were grouped into either SG-1 or severity 
groups 2 and 3. The differential gene expression (DGE) was plotted as an MA-plot, as a measure of the number of genes whose expression was consistently altered between these two groups (Fig. S1C). There were 12 genes (ERG, PROM1, HP, HBD, AHSP, CTSG, PPARG, TIMP4, SMIM10, RNASE1, VSIG4, CMBL, MT1G) that were significantly upregulated in patients in SG-2 and SG-3 compared to SG-1 (Fig. S1C). However, no obvious links between these genes were noted. Serum samples collected during the acute phase of the disease from SG-1 $(n=6)$ and SG-2 $(n=11)$ were analyzed using Luminex assay to assess the soluble cytokine and chemokine markers. Of the 22 markers used for analysis, only IL-8 and GM-CSF were significantly higher in SG-2, and a near significant upregulation was also observed with TNF-a and IL-10 compared to SG-1 (Fig. S2A). The levels of all of the rest 18 biomarkers are shown in Fig. S2B. Cumulatively, the transcriptomics and cytokine array data suggest that there were no significant inflammatory differences with severity in patients who survived CCHF infection.

Transcriptomics changes in the acute phase and post-CCHF recovery. Due to the natural heterogeneity in human cohorts, we used longitudinal samples from 12 patients (SG-1: $n=5 ; S G-2: n=7$ ) to perform differential expression analyses for each infected patient between the time of infection and approx. 1year post-recovery (Range: 46-57 weeks). We observed a clear separation between the acute phase and the recovered phase samples using UMAP clustering (Fig. S3). The DGE profile for the acute phase compared to the recovered phase in all patients showed an upregulation of 2891 genes and a downregulation of 2738 genes (Fig. 1A). The complete profile is given in Supplementary Data File 1. The pathways that are significantly modulated by the upregulated genes (FDR $<0.05)$ are mainly related to metabolic pathways like glycolysis, $\mathrm{N}$-glycan biosynthesis and oxidative phosphorylation (OXPHOS), and antiviral pathways like the NOD-like receptor signaling pathway (Fig. 1B and Supplementary Data File 2). However, the pathways related to the down-regulated genes were mainly related to antiviral defense mechanism-associated pathways including innate immune responses like Th1, Th2, and Th17, cell differentiation, the NF-kB pathways, chemokine signaling pathway, etc. (Fig. 1C). Interestingly, although the HIF-1 signaling pathway was downregulated, the expression of the HIF-1 targeted genes that play a role in glycolysis and coding for the glycolytic enzymes, Enolase 1 (ENO1) and Phosphoglucomutase 2 (PGM2) was significantly upregulated, while hexokinase 1 (HK1) were downregulated (Supplementary Data File 1). However, when we compared the acute phase with the recovered phase in SG-1, and SG-2 separately, there was a distinct differential expression of genes (DEG) profile. In SG-1 the differentially expressed genes were significantly fewer $(n=1617$, upregulated: 954 and downregulated: 663) compared to those in SG-2 ( $n=4256$, upregulated: 2182 and downregulated: 2074) (Fig. 1D and 1E). This indicates that the disease severity had a significant effect on gene expression profiling during the acute phase while comparing when they recovered. There were 1451 overlapping genes between SG-1 and SG-2 that were differentially up-regulated $(n=882)$ and downregulated $(n=569)$. Using gene ontology $(G 0)$ analysis after removal of the redundant terms using $\mathrm{REVIGO}^{\mathbf{1 5}}$, the top two GO terms that were significantly upregulated were the type I interferon signaling pathway (G0:0060337) and the regulation of viral genome replication (GO:0045069) (Fig. 1F). 
Interferon signaling related pathways in CCHFV infection. We further explored the alteration of gene expression in all the interferon signaling related pathways by performing supervised analysis with the genes that are part of $13 \mathrm{GO}$ terms and are part of both type-I and type-II interferon signaling pathways (Fig. 2A). Of the IFN-regulated genes, IFI27 (ISG12) showed the most robust upregulation (Fig. 2B). This was further supported by the RNAscope analysis targeting the IFI27 transcript in the SW13 cell line infected with CCHFV strain IbAr10200 (Fig. 2C). Among the pathways, the maximum significantly differentially expressed genes were part of cellular response to IFN-Y (G0:0071346) $(n=57)$ followed by the Type-I interferon (IFN-I) signaling pathway (GO:0060337) $(n=35)(F i g .2 A)$ and the majority of genes responsible for IFN-I signaling (89\%; 31/35) were upregulated in the acute phase (Fig. 2D). IFI27 has been previously shown to be upregulated specifically during virus infection and has the ability to restrict several viral infections. Apart from this ISG20, ISG15, Mx1, Mx2 and several IFN-a-inducible proteins (IRFs) showed upregulation in the acute phase.

\section{Quantitative temporal proteomics analysis identified modulation of key metabolic process and signaling} pathwaysin vitro. Our longitudinal transcriptomics analysis of CCHF patient samples revealed alternation in the several key metabolic process and signaling pathways during acute phase of infection. To understand the global changes in the host response during CCHFV infection, we infected Huh7 cells with CCHFV and used a time-course proteomic experiment for 24 and $48 \mathrm{hpi}$ using TMT-labelling based massspectrometric analysis (Fig. 3A). The global proteome analysis revealed changes in several pathways that were related to metabolism and the mTOR/HIF-I signaling pathways (Fig. 3B). This was in line with the changes we observed in the patient transcriptomics analysis (Fig. 1B and 1C). Overall, 16 pathways were observed both in patients' transcriptomics and in vitro infection assays in Huh7 cells (Fig. 3B and Fig. S4).

Both our patient data and the in vitro infection model indicated hijacking of the central carbon metabolism (CCM) by CCHFV to meet biosynthetic and bioenergetic demands that could be linked with the Akt/mTOR/HIF-1 pathway (Fig. 4A). To determine the requirement of the CCM and Akt/mTOR/HIF-1 pathway, we blocked glycolysis and glutaminolysis, the two key pathways of CCM in SW13 cells, using 2Deoxy-D-glucose (2-DG) and 6-diazo-5-oxo-L-norleucine (DON) respectively (Fig. 4A) following infection. Infectivity of CCHFV, quantified as relative CCHFV L-gene levels in cells lysates, showed significant decrease in 2-DG treated cells (12-fold, $p<0.0001$ ) and DON treated cells (33-fold, $p<0.0001$ ) (Fig. 4B). We used Akt inhibitor MK2206, to modulate the Akt/mTOR/HIF-1 pathway that we earlier showed effective in blocking SARS-CoV-2 replication in vitro ${ }^{14}$. MK2206 significantly inhibited the CCHFV replication ( 3 -fold, $p<0.0001$ ) to a greater extent. The blocking of the glutaminolysis had maximum effect in blocking the viral replication. It was interesting to note that while 2-DG and DON induced inhibition of viral replication also inhibited the stimulation of CCHFV induced ISGs; ISG15, IFIT1, MX1 and MX2, blocking of viral replication by MK-2206 did not cause any significant reduction in the levels of the ISGs (Fig. 5C).

Temporal dynamics of interferon responsein vitro. To identify the CCHFV induced changes in the interferon protein landscape, we used the GO terms for the cellular response to IFN-y (GO:0071346) and 
the Type-l interferon signaling pathway (GO:0060337) in our time-course proteomic data. The temporal changes in the interferome (cluster of interferon genes) were represented as a heat-map in Fig. 4A. At 24hpi, several ISGs, like Mx1, ISG20 and IFI16, that were upregulated in the acute-phase patient samples were also significantly elevated in infected Huh7 cells (Fig. 4B). Mx1 retained a sustained elevation 48 hpi, and ISG15 was also significantly elevated (Fig. 4C). The most interesting ISG was Mx2 that showed increased mRNA levels $24 \mathrm{hpi}$ in SW13 cells, showed a protein level suppression at $24 \mathrm{hpi}$ and a significant elevation at 48hpi in Huh7 cells (Fig. 4D). We have previously shown the anti-CCHFV role of Mx1 and the interplay between ISG15 and CCHFV was well documented by us ${ }^{16}$ as well as others ${ }^{17}$. Both our transcriptomics and proteomics data from infected patients and infected cell lines respectively, showed induction of ISG20, a 3'-to-5' exonuclease. ISG20 has been reported to have anti-viral activity against several bunyaviruses ${ }^{18}$. To ascertain its effect on CCHFV replication, we used doxycycline inducible stable cell lines that expressed human ISG20 and CAT protein as a control. Following infection with CCHFV at a MOI of 1 for $48 \mathrm{~h}$, there was some degree of inhibition noted in the doxycycline induced ISG20 cell line $(p=0.26)$ and no inhibition was observed in the CAT control cell line (Fig. 4E). Though not significant, the results are suggestive of an antiviral property of ISG20 that is dependent on the enzymatic activity and this requires further investigation. Furthermore, there is also a possibility that the virus has evolved measures to counteract these antiviral responses. During the full course of infection, there was significant suppression of IRF3, the transcription factor for IFN $\beta$ production, indicating viral mediated evasion or delay in activation of the immune response. The time-dependent stimulation of different ISGs by CCHFV suggests differential regulation of the IFN pathway and does not rule out an IFN-I independent mechanism of activation of ISGs.

\section{Discussion}

Metabolic reprogramming during innate antiviral immune responses changes the subsequent phases of disease progression and influences the adaptive immune response. In this study, using the genome-wide transcriptomic analysis of a longitudinal patient cohort, and temporal proteomics from in vitro infection assays in Huh7 cells, we show that during CCHFV infection there is metabolic reprogramming of host cells towards central carbon metabolism that play a central role in viral replication. Blocking the two key central carbon metabolic pathways, glycolysis and glutaminolysis and Akt/mTOR/HIF-1 signaling pathway controlled viral replication in vitro. Moreover, IFN-I mediated antiviral mechanisms were also activated with elevated key antiviral ISGs (ISG12, ISG15, ISG20), and MXs (Mx1 and Mx2).

Both DNA and RNA viruses have been known to alter the host cell metabolism to promote their replication ${ }^{19}$. The transcriptomics data on patient materials and in vitro cell culture assays indicated transient dysregulation of key metabolic processes of the central carbon metabolism, glycolysis, TCAcycle after CCHFV infection, which is known to promote replication in several other RNA viruses including HIV-1, rubella virus, dengue virus, rhinovirus, hepatitis $C$ virus (HCV), influenza virus etc ${ }^{19,} 20,21,22,23$. Blocking glycolysis and glutaminolysis resulted in severe suppression of CCHFV replication suggesting the viral need for these pathways for its efficient replication. The upregulation of Glycolysis in CCHFV 
infection could be mediated by Akt/mTOR/HIF pathway, as blocking Akt/mTOR signaling following infection showed dramatic decrease in viral replication and furthermore in our previous study we have shown that exogenous nitric oxide that is known to regulate the HIF-1 via the Akt/mTOR pathway under normoxic conditions ${ }^{24}$, inhibited CCHFV in vitro ${ }^{25}$. However, in our patient transcriptomics data we also noted significant downregulation of genes belonging to the PI3K/Akt pathway during acute CCHFV infection, indicating the dysregulation of a pathway that is critical for the cellular defense against invading pathogens. Interestingly, an in vitro study in another Bunyaviridae virus, Rift valley fever virus (RVFV), identified the inhibition of the PI3K/Akt pathway by dephosphorylation of the AKT and Forkhead box protein 01 (Fox01) ${ }^{26}$. In our study we observed that the majority of the coding genes in the FoxO signaling pathway were downregulated by CCHFV, including the FOXO transcription factors FOXO1 and $\mathrm{FOXO3}$, that can act as negative feedback regulators of the innate cellular antiviral response ${ }^{27}$. FoxO1 and FoxO3 also play an essential role in the immunometabolic dynamics and are important targets for glycolysis and gluconeogenesis ${ }^{28,29}$. Another critical signaling pathway that was downregulated during the CCHFV infection was AMP-activated protein kinase (AMPK) signaling that has been known to support IFN-I production through metabolic reprogramming. ${ }^{30}$

In recent years, it has been recognized that metabolic pathways play a role in mounting effective IFN-I response $^{31}$ and the mechanisms are still not well understood. We observed a marked inhibition of CCHFV infection induced ISGs like ISG15, IFIT1, MX1 and MX2 mRNA levels upon blocking glycolysis and glutaminolysis. This could be either due to inhibition of CCHFV replication resulting in reduced replication products that could not elicit type-I IFN signaling pathways or blocking of glycolysis and glutaminolysis did not meet the energy requirement for successful progression of the IFN signaling cascades. Interestingly the inhibition of CCHFV replication by MK2206 that blocks Akt did not cause significant reduction in ISGs mRNA. Akt has been shown to regulate mRNA translation of ISGs ${ }^{32}$ and thus its effect on the mRNA levels may not be profound and in this study, we did not determine the functionality of the IFN response. However, ability of the CCHFV virus to induce ISG expression in absence of viral replication under Akt inhibition suggests towards the activation of IFN-signaling cascades and importance of CCM in regulation of IFN-I pathways during viral infection.

The interferon signaling pathways play a central role in innate immune responses against viruses and mediates stimulation of antiviral ISGs and secretion of pro-inflammatory cytokines. The protective role of IFN-I against CCHFV has been exemplified in mouse models, in which IFNAR ${ }^{-/-}$or STAT-1 ${ }^{-/-}$mice $^{33,34}$ or $\mathrm{STAT}^{-/-}$hamsters ${ }^{35}$ showed enhanced susceptibility to CCHFV infection. Even in in-vitro experiments, pre-treatment of cells with IFN-a was found to be inhibitory to CCHFV ${ }^{12}$. Though CCHFV is inhibited by the IFN -response, not many ISGs with anti CCHFV activity have been identified apart from MxA although, ISG20 and PKR have been proposed ${ }^{16}$ to have anti-CCHFV activity. In the present study we observed that several ISGs with known or proposed anti-CCHFV activity, i.e. Mx1, ISG15 and ISG20 or not defined for CCHFV like IFIT3, IFITM3, IFI16 and OAS3 were upregulated in the acute phase CCHFV patient samples as well as in the cell-infection model. Among these, ISG20 has been shown to have antiviral activity against 
related-Bunyaviruses ${ }^{18}$, and we observed some degree of inhibitory activity of ISG20 on CCHFV replication.

Like IFN-I, proinflammatory cytokines are important for effective innate immunity. CCHFV patients are known to have higher levels of plasma cytokine and chemokine compared to healthy people. Elevated levels of IL-6, IL-10 and TNF- $a$ are most commonly associated with fatal outcomes ${ }^{36,37,38}$. A few studies have suggested that the co-elevation of IL- 6 and TNF-a are strongly associated with fatality ${ }^{38}$. Our study did not include samples from fatal cases and thus we performed a cytokine array and compared moderate and severe cases. Our results further confirmed the roles of TNF-a, IL-10, IL-8 and GM-CSF since these showed significant or near-significant elevation in severe cases. Korva et al., also observed significant differences in IL-10 and GM-CSF between moderate and severe cases ${ }^{36}$. In another study by Hulva et al., changes in IL-6 and TNF- $a$ were noted between moderate and severe cases, but no significant change in IL-8 was observed ${ }^{39}$. Although IL- 6 is often associated with disease severity and fatality, we did not observe any significant difference in IL-6 levels.

In conclusion, our study provides a comprehensive description of the host-immune response against CCHFV that can explain viral pathogenesis. The interplay of the metabolic reprogramming and the IFNmediated host antiviral mechanism could provide attractive options for therapeutic intervention of CCHF. The specific role of the different ISGs that were regulated during the course of infections need to be studied further to provide important clues for the development of antivirals or vaccines.

\section{Materials And Methods}

\section{Ethics statement and biosafety}

This study was approved by the Local Research Ethics Committee of the Ankara Numune Education and Research Hospital, Turkey (Protocol \# 17-1338) and Regional Ethics Committee, Stockholm (Dnr. 2017-/1712-31/2). All patients and/or their relatives were informed about the purpose of the study and signed a consent form before collection.

\section{Study design, patients, and sample collection:}

We enrolled 18 adult patients ( $\geqq 18$ years) diagnosed with CCHF who were followed up by the clinical service of Infectious Diseases and Clinical Microbiology of Sivas Cumhuriyet University Hospital, Sivas, Turkey. The CCHF patients were divided into three groups using the SGS scores of 1,2 and $3{ }^{40}$. Blood samples were collected on the admission day (acute stage) and from the survivors one year after their recovery (Supplementary Table 1) following confirmed positive real-time RT-PCR test (Altona Diagnostics ${ }^{\circledR}$, Hamburg, Germany) and/or serology by lgM indirect immunofluorescence antibody (IFA) assay (Euroimmun ${ }^{\circledR}$, Luebeck, Germany). Peripheral blood mononuclear cells (PBMCs) and plasma were sent to the Public Health Agency of Sweden, Stockholm, Sweden. Serum cytokine profiling targeting 22 cytokines/chemokines was performed by Public Health England using methods as previously described 
${ }^{41}$ using a 22 -plex customised luminex kit (Merck Millipore, Darmstadt, Germany) was designed to quantitate: Eotaxin, Granulocyte-Colony Stimulating Factor (G-CSF), Granulocyte Macrophage-Colony Stimulating Factor (GM-CSF), Interferon-alpha 2 (IFN-a2), Interferon-gamma (IFN- - ), Interleukin (IL)-10, IL12, IL-15, IL-17a, IL-1a, IL-9, IL-1b, IL-2, IL-4, IL-5, IL-6, IL-8, IFN-y-Inducible Protein 10 (IP-10), Monocyte Chemotactic Protein 1 (MCP-1), Macrophage Inhibitory Protein (MIP)-1a, MIP-1b and Tumour Necrosis Factor alpha (TNF-a). Two quality control samples and a standard included with the kit were prepared as per the manufacturer's instructions. A Luminex MAGPIX instrument using Exponent software (Invitrogen, Paisley, UK) was used to obtain results in the form of MFI (median fluorescence intensity), which was converted to concentration $(\mathrm{pg} / \mathrm{ml})$ using the standard curve prepared. Plate data was accepted based on negative background wells and QC data within the expected ranges.

\section{RNA extraction and sequencing (Illumina RNAseq) and transcriptomics analysis:}

Total RNA was extracted from Trizol-treated PBMC using the Direct-zol RNA Miniprep (Zymo Research, CA, USA) according to the manufacturer's protocol. RNA-Seq was performed at the National Genomics Infrastructure, Science for Life Laboratory, Stockholm, Sweden, as described by us previously ${ }^{42}$. The transcriptomics data analysis was performed as described by us recently ${ }^{14}$. The distribution of all samples was then visualized after reducing the dimension of the data by applying the Uniform Manifold Approximation and Projection for Dimension Reduction (UMAP) technique using $\mathrm{R}$ package umap v0.2.6. ${ }^{43}$. The reduced dimensions of the data were plotted in 2D space using $\mathrm{R}$ package ggplot2 v3.3.2 $2^{44}$. Differential gene expression analysis was performed using raw read counts of remaining samples using the R/Bioconductor package DESeq2 v1.26.045. Genes with adjusted p-values of less than 0.05 were considered as significantly regulated. Functional analysis of the significantly regulated genes was performed using the enrichr module of the python package GSEAPY v 0.9.16 46,47 . Gene ontology (GO) enrichment analysis was performed using the Enrichr web resource ${ }^{47}$, and the GO biological process 2018 category was selected. Redundant GO terms were removed using the online tool REVIGO ${ }^{15}$. Heatmaps were generated using the R/Bioconductor package ComplexHeatmap v2.2.048. Bubble plots, MA plots, Volcanoplots, violin plots and bar plots were created using R package ggplot2 v3.3.2. Network visualization was performed using Cytoscape v3.6.1 $1^{49}$. Venn diagrams were constructed using the online tool InteractiVenn ${ }^{50}$. All the codes are available in GitHub (https://neogilab.github.io/CCHF-Turkey/). Raw RNAseq data is available in Sequence Read Archive (SRA) with temporary id SUB8608640.

\section{Cells and viruses:}

The CCHFV strain IbAr10200 (originally isolated from Hyalomma excavatum ticks from Sokoto, Nigeria, in 1966) was used in this study. The SW13- $A T C C^{\circledR}-C C L-105^{T M}$ and human hepatocyte-derived cellular carcinoma cell line Huh7 was obtained from Marburg Virology Laboratory, (Philipps-Universität Marburg, Marburg, Germany) and matched the STR reference profile of Huh $7^{51}$. CCHFV was propagated and titered in SW13 cells. The virus was serially diluted 10 -fold and 100ul of each dilution was added to SW13 cells in a 96 well plate. At $48 \mathrm{~h}$ post-infection (hpi), cells were fixed in ice-cold acetone-methanol (1:1) and 
stained using a rabbit polyclonal anti-CCHFV nucleocapsid antibody followed by a fluorescein isothiocyanate (FITC)-conjugated anti-rabbit antibody (Thermo Fisher Scientific, US) and DAPI (Roche, US). The number of fluorescent foci in each well was counted using a fluorescence microscope and the titer was determined. Human 293 FLP-IN TRex cells expressing CAT or ISG20 under the control of a Tet-on promoter were kindly provided by Ju-Tao Guo (Drexel University College of Medicine, Pennsylvania, USA). ${ }^{52}$ The cells were propagated in DMEM supplemented with $5 \%$ Tet-negative FCS (PAA Lab, US).

\section{In vitro infection assay in Huh7 cells and tandem mass tag (TMTpro) labelled reverse phase liquid chromatography mass-spectrometric (RPLC-MS/MS) analysis:}

Huh7 cells were infected with the CCHFV in triplicate, as described by us previously ${ }^{14}$. Briefly, Huh7 cells were infected with CCHFV IbAr10200 at a multiplicity of infection (MOI) of 1 . After $1 \mathrm{~h}$ of incubation $\left(37^{\circ} \mathrm{C}, 5 \% \mathrm{CO}_{2}\right)$ the inoculum was removed, the cells were washed with PBS, and $2 \mathrm{ml} \mathrm{DMEM}$ supplemented with $5 \%$ heat-inactivated FBS was added to each well. Samples were collected in triplicate at 24 and $48 \mathrm{hpi}$ along with controls. Following the protein digestion in S-Trap microcolumns (Protifi, Huntington, NY), the resulting peptides were labeled with TMTpro tags. Labeled peptides were fractionated by high $\mathrm{pH}(\mathrm{HpH})$ reversed-phase chromatography, and each fraction was analyzed on an Ultimate 3000 UHPLC (Thermo Scientific, San Jose, CA) in a 120 min linear gradient. Proteins were searched against the SwissProt human database using the search engine Mascot v2.5.1 (MatrixScience Ltd, UK) in Proteome Discoverer v2.4 (Thermo Scientific) software allowing up to two missed cleavages.

\section{Proteomics data analysis:}

The raw data were first filtered to remove missing data. Proteins detected in all samples were retained for analysis resulting in 8,501 proteins in the filtered dataset. The filtered data was then normalized by applying eight different methods using R/Bioconductor package NormalyzerDE v1.4.0 ${ }^{53}$. The quantile normalization was found superior to other methods and was selected for further use. Differential protein expression analysis was performed using R/Bioconductor package limma v3.42.2 ${ }^{54}$. Proteins with adjusted $p$-values of less than 0.05 were regarded as significant. KEGG pathway enrichment analysis of significantly regulated proteins was performed using the enrichr module of python package GSEAPY $v$ 0.9.16. The mass spectrometry proteomics data have been deposited to the ProteomeXchange Consortium via the PRIDE partner repository with the dataset identifier PXD022672.

\section{ISG20 inhibition assay:}

Tet-inducible HEK-293 FLP-IN TRex cells containing a CAT or ISG20 expression cassette were seeded on in six-well dishes and treated with doxycycline at a final concentration of $2 \mu \mathrm{g} / \mathrm{ml}$ and incubated for $36 \mathrm{~h}$. Cells were infected with CCHFV strain IbAr10200 at an MOI of 1, and supernatants were titrated at 48 hpi by enumeration of fluorescent foci and calculation of progeny virus titers as described by us previously ${ }^{11}$. 
The RNAscope ${ }^{\circledR}$ ISH Assays (ACD Bioscience,US) targeting IFI27 (440111, ACD Bioscience, US) and CCHFV (510621, ACD Bioscience, US) were perfomed as described previously ${ }^{55}$. SW13 cells were infected with CCHFV (Ibar 10200 strain) at MOI of 0.1. After 1h, inoculum was replenished with fresh Leitbovitz medium containing $5 \%$ FBS and incubated $48 \mathrm{~h}$. After infection cells were fixed 30 min using ice-cold acetone. Cells were permeabilized using $0.1 \%$ Triton $x-100$ for 10 min at RT prior probe incubation at $40^{\circ} \mathrm{C}$ of IFI27 and CCHFV probes (RNAscope® ISH ACD,US) for 2h, 30min, $15 \mathrm{~min}, 30 \mathrm{~min}$ and $15 \mathrm{~min}$, respectively. Cells were further stained using DAPI and coverslip attached using ProLong Gold Antifade reagent (P10144, Thermofisher). Images were acquired using Nikon Single Point scanning confocal with $60 / 1.4$ oil objective.

\section{Metabolic perturbation and virus infection.}

To inhibit glycolysis, glutaminolysis and Akt/mTOR pathway, following $1 \mathrm{hpi}$ (moi 0.1 ) the cells were treated with 2-deoxy-D-glucose (2-DG, 5mM), diazo-5-oxo-L-norleucine (DON, 50mM) and MK-2206 $(10 \mathrm{mM})$ respectively. The cells were collected after $24 \mathrm{hpi}$ and the cells were lysed in Trizol reagent. RNA was extracted using the Direct-zol RNA Miniprep kit (Zymo Research, Irvine, CA) according to the manufacturer's instructions. Viral RNA was measured by quantitative real real-time polymerase chain reaction (qRT-PCR) using TaqMan Fast Virus 1-Step Master Mix (Thermo Fisher Scientific) with primers and probe specific for the CCHFV L gene; Forward: 5-GCCAACTGTGACKGTKTTCTAYATGCT-3', Reverse-1: 5'- CGGAAAGCCTATAAAACCTACCTTC-3', Reverse-2: 5'-CGGAAAGCCTATAAAACCTGCCYTC-3' and Reverse-3: 5'-CGGAAAGCCTAAAAAATCTGCCTTC-3' and Probe FAM-CTGACAAGYTCAGCAAC -MGB. RNAse was used as endogenous control. The cycling reactions was performed using a capillary Roche LightCycler 2.0 system.

Messanger RNA (mRNA) expression of a few ISG transcripts (ISG15, IFIT1, MX1 and MX2) and human bactin were measured by qRT-PCR. The sequences of the qPCR primers are listed in supplemental table S1. Reverse transcription of extracted RNA was performed using a high capacity reverse transcription kit (Applied Biosystems, USA) for $10 \mathrm{~min}$ at $25^{\circ} \mathrm{C}$, followed by $37^{\circ} \mathrm{C}$ for $120 \mathrm{~min}$ and $85^{\circ} \mathrm{C}$ for $5 \mathrm{~min}$. Quantitative RT-PCR assays were setup using the Power SYBR Green PCR Master Mix (Applied Biosystems, UK) using $250 \mathrm{nM}$ of primer pairs with cycling conditions: initial denaturation $95^{\circ} \mathrm{C}$ for 10 min, followed by 40 cycles of $95^{\circ} \mathrm{C}$ for $15 \mathrm{sec}, 60^{\circ} \mathrm{C}$ for $1 \mathrm{~min}$. Melting curves were run by incubating the reaction mixtures at $95^{\circ} \mathrm{C}$ for $15 \mathrm{sec}, 60^{\circ} \mathrm{C}$ for $20 \mathrm{sec}, 95^{\circ} \mathrm{C}$ for $15 \mathrm{sec}$, ramping from $60^{\circ} \mathrm{C}$ to $95^{\circ} \mathrm{C}$ in $1^{\circ} \mathrm{C} / \mathrm{sec}$. The values were normalized to endogenous $\mathrm{b}$-actin. Fold change was calculated as: Fold Change $=2-\Delta(\Delta \mathrm{Ct})$.

\section{Declarations}

Acknowledgments: The authors would like to acknowledge support from the National Genomics Infrastructure (NGI), Science for Life Laboratory, for RNAseq and Proteomics Biomedicum; and Karolinska Institute, Solna, for LC-MS/MS analysis. The computations were performed using resources provided by SNIC through the Uppsala Multidisciplinary Center for Advanced Computational Science (UPPMAX) under 
Project SNIC2017-550. The microscopy part of the study was performed at the Live Cell Imaging Facility, Karolinska Institute, Sweden, supported by grants from the Knut and Alice Wallenberg Foundation, the Swedish Research Council, the Centre for Innovative Medicine, and the Jonasson Center at the Royal Institute of Technology, Sweden. The study is funded by Swedish Research Council Grants 2018-05766 and 2017-03126 to AM and 2017-01330 to UN, PHE Grant In Aid 109509 (inc. PhD studentship[EK]) and EU-H2020 CCHFVaccine to RH.

Author contributions: Conceptualization: U.N. and A.M., Methodology: U.N. N.E. A.M., A.V., F.W., and R.H., Formal analysis: A.T.A., S.A.,E.K., S.D., B.B., S.G., J.E.R, V.M.M. and S.S., Resources: U.N., A.M., F.W., and R.H., Writing (original draft): U.N., A.T.A, and S.G., Writing (review and editing): N.E., S.A., A.V., F.W., R.H., and A.M., Visualization: U.N., A.T.A, and S.S., Supervision: U.N., A.M., A.V., N.E., F.W., R.H., Project administration: U.N., N.E. and A.M., Funding acquisition: U.N., A.M., F.W., and R.H. All authors discussed the results, commented, and approved the final version of the manuscript.

Declaration of interests: The authors declare no competing interests.

\section{References}

1. WHO. Fact Sheet: Crimean Congo Haemorrhagic Fever.). World Health Organization Regional Office for Africa (2020).

2. Bente DA, Forrester NL, Watts DM, McAuley AJ, Whitehouse CA, Bray M. Crimean-Congo hemorrhagic fever: history, epidemiology, pathogenesis, clinical syndrome and genetic diversity. Antiviral Res 100, 159-189 (2013).

3. Zivcec M, Scholte FE, Spiropoulou CF, Spengler JR, Bergeron É. Molecular Insights into CrimeanCongo Hemorrhagic Fever Virus. Viruses 8, 106 (2016).

4. ECDC. Hyalomma marginatum - Factsheet for experts, European Centre for Disease Prevention and Control.) (2020).

5. Grandi G, et al. First records of adult Hyalomma marginatum and H. rufipes ticks (Acari: Ixodidae) in Sweden. Ticks Tick Borne Dis 11, 101403 (2020).

6. McGinley L, et al. First report of human exposure to Hyalomma marginatum in England: Further evidence of a Hyalomma moulting event in north-western Europe? Ticks Tick Borne Dis 12, 101541 (2020).

7. Estrada-Peña A, Sánchez N, Estrada-Sánchez A. An assessment of the distribution and spread of the tick Hyalomma marginatum in the western Palearctic under different climate scenarios. Vector Borne Zoonotic Dis 12, 758-768 (2012).

8. Yu Y, Clippinger AJ, Alwine JC. Viral effects on metabolism: changes in glucose and glutamine utilization during human cytomegalovirus infection. Trends Microbio/ 19, 360-367 (2011).

9. Aller S, Scott A, Sarkar-Tyson M, Soyer OS. Integrated human-virus metabolic stoichiometric modelling predicts host-based antiviral targets against Chikungunya, Dengue and Zika viruses. $J R$ 
Soc Interface 15, (2018).

10. Molenaar D, van Berlo R, de Ridder D, Teusink B. Shifts in growth strategies reflect tradeoffs in cellular economics. Mol Syst Biol 5, 323 (2009).

11. Andersson I, Lundkvist A, Haller O, Mirazimi A. Type I interferon inhibits Crimean-Congo hemorrhagic fever virus in human target cells. J Med Virol 78, 216-222 (2006).

12. Andersson I, Karlberg H, Mousavi-Jazi M, Martínez-Sobrido L, Weber F, Mirazimi A. Crimean-Congo hemorrhagic fever virus delays activation of the innate immune response. J Med Viro/ 80, 13971404 (2008).

13. Fritsch SD, Weichhart T. Effects of Interferons and Viruses on Metabolism. Front Immuno/ 7, 630 (2016).

14. Appelberg $\mathrm{S}$, et al. Dysregulation in Akt/mTOR/HIF-1 signaling identified by proteo-transcriptomics of SARS-CoV-2 infected cells. Emerg Microbes Infect 9, 1748-1760 (2020).

15. Supek F, Bošnjak M, Škunca N, Šmuc T. REVIGO summarizes and visualizes long lists of gene ontology terms. PLoS One 6, e21800 (2011).

16. Andersson I, et al. Human MxA protein inhibits the replication of Crimean-Congo hemorrhagic fever virus. J Virol 78, 4323-4329 (2004).

17. Scholte FEM, et al. Crimean-Congo Hemorrhagic Fever Virus Suppresses Innate Immune Responses via a Ubiquitin and ISG15 Specific Protease. Cell Rep 20, 2396-2407 (2017).

18. Feng J, et al. Interferon-Stimulated Gene (ISG)-Expression Screening Reveals the Specific Antibunyaviral Activity of ISG20. J Viro/ 92, (2018).

19. Mayer KA, Stöckl J, Zlabinger GJ, Gualdoni GA. Hijacking the Supplies: Metabolism as a Novel Facet of Virus-Host Interaction. Front Immunol 10, 1533 (2019).

20. Smallwood HS, et al. Targeting Metabolic Reprogramming by Influenza Infection for Therapeutic Intervention. Cell Rep 19, 1640-1653 (2017).

21. Eisenreich W, Rudel T, Heesemann J, Goebel W. How Viral and Intracellular Bacterial Pathogens Reprogram the Metabolism of Host Cells to Allow Their Intracellular Replication. Front Cell Infect Microbio/ 9, 42 (2019).

22. Thaker SK, Ch'ng J, Christofk HR. Viral hijacking of cellular metabolism. BMC Biol 17, 59 (2019).

23. Fontaine KA, Sanchez EL, Camarda R, Lagunoff M. Dengue virus induces and requires glycolysis for optimal replication. J Viro/ 89, 2358-2366 (2015).

24. Sandau KB, Faus HG, Brüne B. Induction of hypoxia-inducible-factor 1 by nitric oxide is mediated via the PI 3K pathway. Biochem Biophys Res Commun 278, 263-267 (2000).

25. Simon M, Falk KI, Lundkvist A, Mirazimi A. Exogenous nitric oxide inhibits Crimean Congo hemorrhagic fever virus. Virus Res 120, 184-190 (2006).

26. Popova TG, et al. Reverse-phase phosphoproteome analysis of signaling pathways induced by Rift valley fever virus in human small airway epithelial cells. PLoS One 5, e13805 (2010). 
27. Lei CQ, Zhang Y, Xia T, Jiang LQ, Zhong B, Shu HB. FoxO1 negatively regulates cellular antiviral response by promoting degradation of IRF3. J Biol Chem 288, 12596-12604 (2013).

28. Puigserver $\mathrm{P}$, et al. Insulin-regulated hepatic gluconeogenesis through FOX01-PGC-1alpha interaction. Nature 423, 550-555 (2003).

29. Lundell LS, Massart J, Altıntaş A, Krook A, Zierath JR. Regulation of glucose uptake and inflammation markers by FOXO1 and FOXO3 in skeletal muscle. Mol Metab 20, 79-88 (2019).

30. Hurley $\mathrm{HJ}$, et al. Frontline Science: AMPK regulates metabolic reprogramming necessary for interferon production in human plasmacytoid dendritic cells. J Leukoc Biol 109, 299-308 (2021).

31. Ahmed D, Cassol E. Role of cellular metabolism in regulating type I interferon responses: Implications for tumour immunology and treatment. Cancer Lett 409, 20-29 (2017).

32. Kaur $\mathrm{S}$, et al. Role of the Akt pathway in mRNA translation of interferon-stimulated genes. Proc Natl Acad Sci U S A 105, 4808-4813 (2008).

33. Bente DA, et al. Pathogenesis and immune response of Crimean-Congo hemorrhagic fever virus in a STAT-1 knockout mouse model. J Viro/ 84, 11089-11100 (2010).

34. Zivcec M, Safronetz D, Scott D, Robertson S, Ebihara H, Feldmann H. Lethal Crimean-Congo hemorrhagic fever virus infection in interferon $\alpha / \beta$ receptor knockout mice is associated with high viral loads, proinflammatory responses, and coagulopathy. J Infect Dis 207, 1909-1921 (2013).

35. Ranadheera $\mathrm{C}$, et al. Characterization of a novel STAT 2 knock-out hamster model of Crimean-Congo hemorrhagic fever virus pathogenesis. Sci Rep 10, 12378 (2020).

36. Korva M, et al. Characterization of Biomarker Levels in Crimean-Congo Hemorrhagic Fever and Hantavirus Fever with Renal Syndrome. Viruses 11, (2019).

37. Saksida A, Duh D, Wraber B, Dedushaj I, Ahmeti S, Avsic-Zupanc T. Interacting roles of immune mechanisms and viral load in the pathogenesis of crimean-congo hemorrhagic fever. Clin Vaccine Immunol 17, 1086-1093 (2010).

38. Ergonul O, Tuncbilek S, Baykam N, Celikbas A, Dokuzoguz B. Evaluation of serum levels of interleukin (IL)-6, IL-10, and tumor necrosis factor-alpha in patients with Crimean-Congo hemorrhagic fever. $J$ Infect Dis 193, 941-944 (2006).

39. Hülya Y, Yilmaz G, Kostakoglu U, Arslan M, Karahan SC, Koksal I. Evaluation of Serum TNF-alpha, IL6, IL-10, and IFN-gamma Levels in Patients with Crimean-Congo Hemorrhagic Fever. Open Forum Infectious Diseases 4, S312-S313 (2017).

40. Bakir M, Engin A, Gozel MG, Elaldi N, Kilickap S, Cinar Z. A new perspective to determine the severity of cases with Crimean-Congo hemorrhagic fever. J Vector Borne Dis 49, 105-110 (2012).

41. Dowall SD, Graham VA, Fletcher T, Hewson R. Use and reliability if multiplex bead-based assays (Luminex) at Containment Level 4. Methods 158, 17-21 (2019).

42. Zhang W, et al. Transcriptomics and Targeted Proteomics Analysis to Gain Insights Into the Immunecontrol Mechanisms of HIV-1 Infected Elite Controllers. EBioMedicine 27, 40-50 (2018). 
43. Leland M, John H, Nathaniel S, Lukas G. UMAP: uniform manifold approximation and projection. Journal of Open Source Software 3, 861 (2018).

44. Wickham H. ggplot2: Elegant Graphics for Data Analysis. Springer International Publishing (2009).

45. Love MI, Huber W, Anders S. Moderated estimation of fold change and dispersion for RNA-seq data with DESeq2. Genome Biol 15, 550 (2014).

46. Subramanian A, et al. Gene set enrichment analysis: a knowledge-based approach for interpreting genome-wide expression profiles. Proc Natl Acad Sci U S A 102, 15545-15550 (2005).

47. Kuleshov MV, et al. Enrichr: a comprehensive gene set enrichment analysis web server 2016 update. Nucleic Acids Res 44, W90-97 (2016).

48. Gu Z, Eils R, Schlesner M. Complex heatmaps reveal patterns and correlations in multidimensional genomic data. Bioinformatics 32, 2847-2849 (2016).

49. Shannon P, et al. Cytoscape: a software environment for integrated models of biomolecular interaction networks. Genome Res 13, 2498-2504 (2003).

50. Heberle H, Meirelles GV, da Silva FR, Telles GP, Minghim R. InteractiVenn: a web-based tool for the analysis of sets through Venn diagrams. BMC Bioinformatics 16, 169 (2015).

51. Rohde C, Becker S, Krahling V. Marburg virus regulates the IRE1/XBP1-dependent unfolded protein response to ensure efficient viral replication. Emerg Microbes Infect 8, 1300-1313 (2019).

52. Jiang $D$, et al. Identification of five interferon-induced cellular proteins that inhibit west nile virus and dengue virus infections. J Virol 84, 8332-8341 (2010).

53. Willforss J, Chawade A, Levander F. NormalyzerDE: Online Tool for Improved Normalization of Omics Expression Data and High-Sensitivity Differential Expression Analysis. J Proteome Res 18, 732-740 (2019).

54. Ritchie ME, et al. limma powers differential expression analyses for RNA-sequencing and microarray studies. Nucleic Acids Res 43, e47 (2015).

55. Zhang W, Svensson Akusjarvi S, Sonnerborg A, Neogi U. Characterization of Inducible Transcription and Translation-Competent HIV-1 Using the RNAscope ISH Technology at a Single-Cell Resolution. Front Microbio/ 9, 2358 (2018).

\section{Supplementary Materials}

Supplementary Figure S1: Severity group association with gene expression. (A) Sample distribution during the acute phase of infection in different severity groups as reported. (B) The heterogenous geneexpression profile was further refined by hierarchical clustering analysis of the top 500 high variable genes (C) MA plot of differentially regulated genes during the acute phase between samples of SG-1 and SG-2 or 3 .

Supplementary Figure S2. (A) Violin plot of significantly changed proteins as determined from ELISA assays. (B) The levels of all of the rest 18 soluble marker. 
Supplementary Fig. S3: UMAP clustering of the acute phase and recovered phase samples.

Supplementary Fig. S4: Overlap of the pathways identified between patients-based transcriptomics data and in vitro cellular data.

Supplementary Fig. S5. Potential pathways regulated by CCHFV during acute phase of infection

Supplementary Data File 1: The DGE profile for the acute phase compared to the recovered phase in all patients showed an upregulation of 2891 genes and a downregulation of 2738

Supplementary Data File 2: The pathways that are significantly modulated by the upregulated and downregulated genes.

\section{Figures}

A.

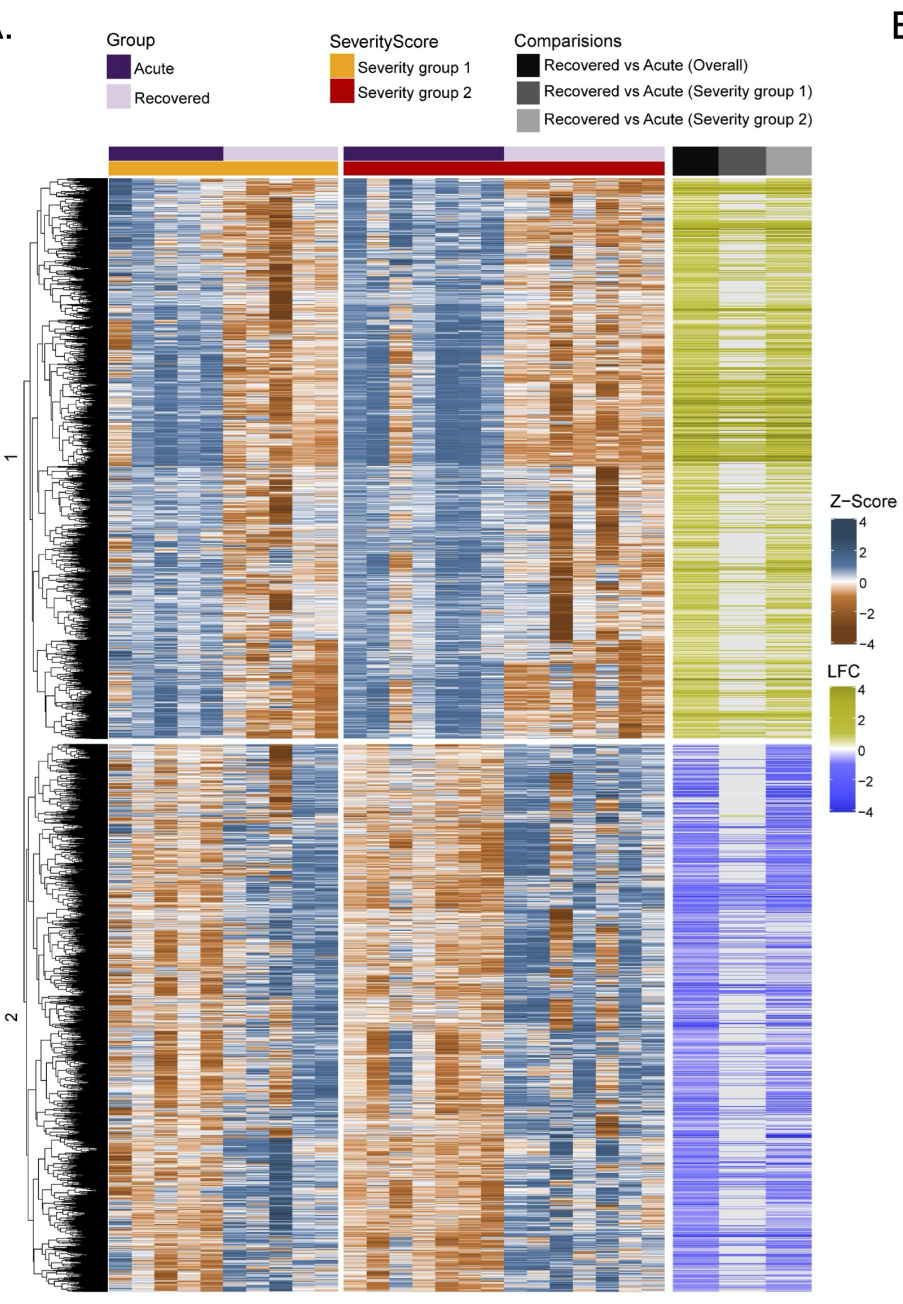

B.

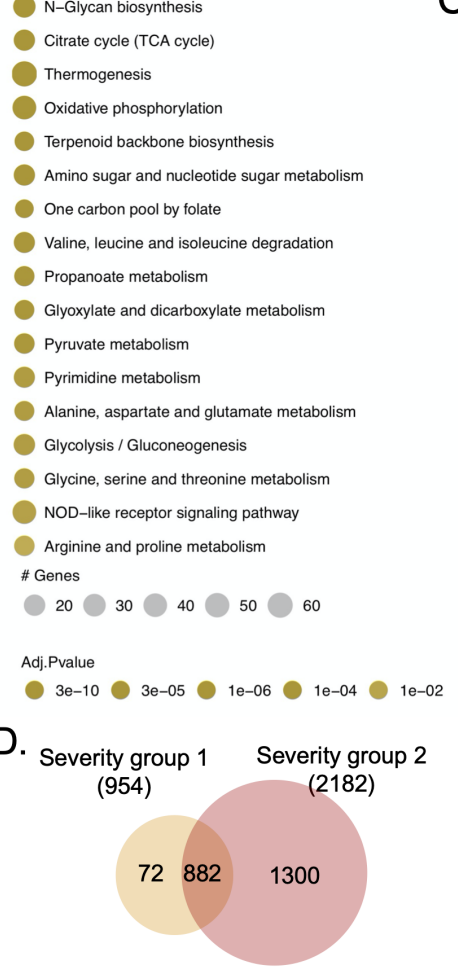

F.

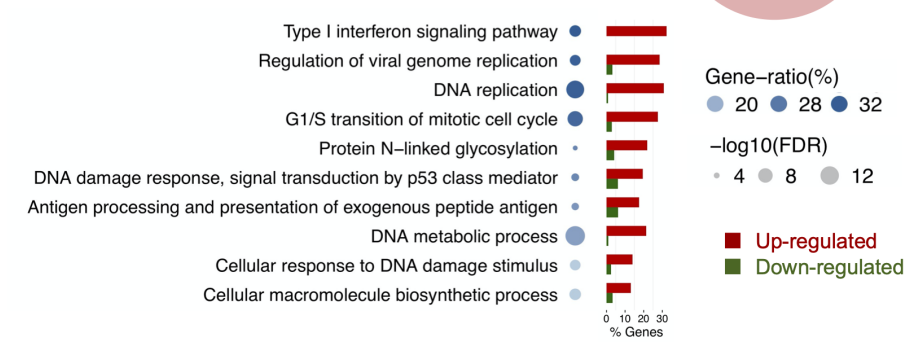

C.

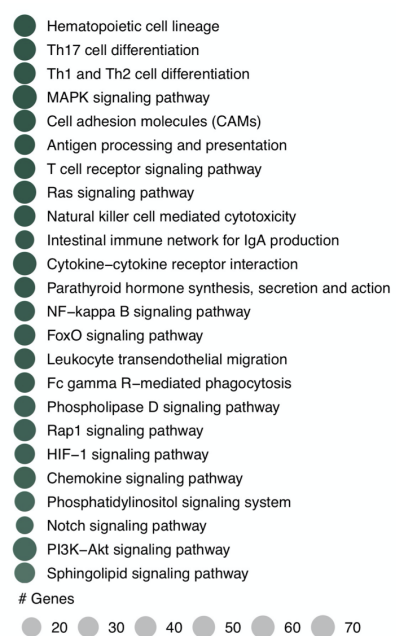

$20 \bigcirc 30 \bigcirc 40 \bigcirc 50 \bigcirc 60 \bigcirc 70$

Adj.Pvalue

3e-10 $3 \mathrm{e}-05 \bigcirc 1 \mathrm{C}-05 \bigcirc 1 \mathrm{e}-04 \bigcirc 1 \mathrm{e}-02$

E.

Severity group $1 \quad$ Severity group 2

(663)

(2074)

\section{Figure 1}

Differential gene expression and pathway analysis between acute and recovery phases. (A) Heatmap of Z-score transformed expression values of significantly regulated genes in the pair-wise comparisons 
namely Recovered vs Acute (overall), Recovered vs Acute (SG-1), Recovered vs Acute (SG-2). The columns represent the patient samples and their corresponding severity groups at different time points. The rows represent genes that are hierarchically clustered based on Euclidean distance. (B) KEGG pathway enrichment analysis results of genes belonging to cluster 1 of the heatmap. The bubble size and colour gradient correspond to the number of genes and adjusted $P$ value of the enrichment test, respectively. (C) KEGG pathway enrichment results of genes belonging to cluster 2 of the heatmap. The bubble size and color gradient correspond to the number of genes and adjusted $P$ value of the enrichment test, respectively. (D) Venn diagram of significantly up-regulated genes in Recovered vs Acute (SG-1) and Recovered vs Acute (SG-2) phases (E) Venn diagram of significantly down-regulated genes in Recovered vs Acute (SG-1) and Recovered vs Acute (SG-2) phases. (F) Gene ontology (biological process) enrichment analysis results of commonly regulated genes (882 upregulated and 569 down-regulated) from (d) and (e). the colour gradient and bubble size correspond to the gene ratio of each GO term and the adjusted $P$ value of the enrichment test, respectively. The adjacent bar graph represents the percentage of genes upregulated or downregulated in each gene ontology term. 


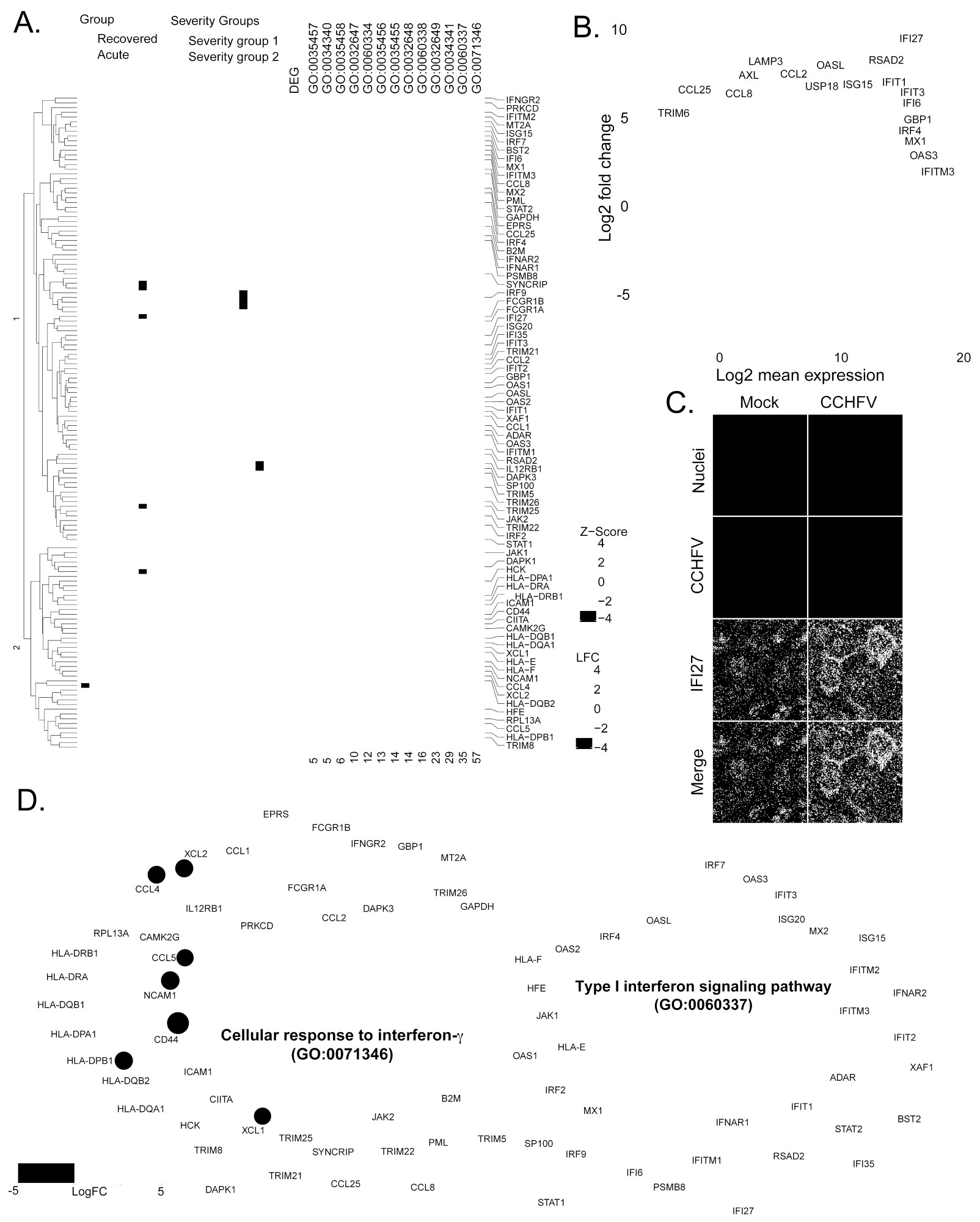

Figure 2

Differentially expressed genes in interferon (IFN) signaling pathways. (A) Heatmap visualizing the expression pattern of IFN-signaling genes (including ISGs) that are significantly different between the recovered and acute phases. The columns represent the patient samples and their corresponding severity groups at different time points. The rows represent genes hierarchically clustered based on Euclidean distance. The row annotation indicates the differential expression of genes (DEG) and presence or 

(G0:0071346) and the IFN-Isignaling (G0:0060337) are printed. (B) MA plot of differentially regulated genes between the recovered and acute phases. ISGs are marked. (C) RNAscope analysis targeting IFI27 genes in infected and non-infected cells. (D) Network type visualization of genes belonging to G0:0071346 and G0:0060337. The edges indicate the presence of the corresponding gene in the geneontology term. The node size and color gradient correspond to the adjusted $\mathrm{P}$ value of differential expression analysis and the log2 fold change, respectively.

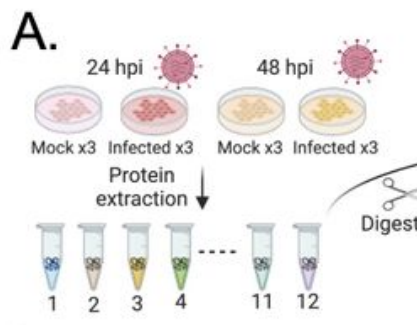

B.

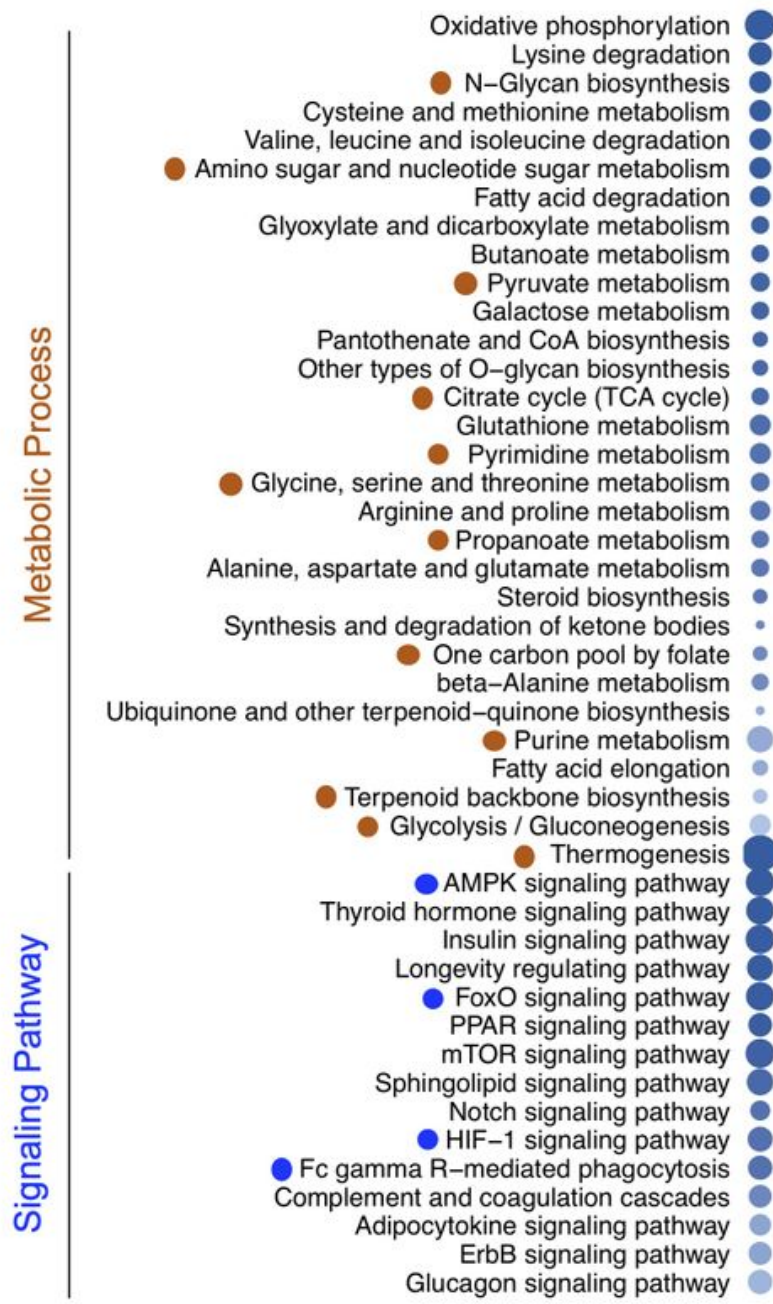

Changes over time (24hpi vs $48 \mathrm{hpi}$ )

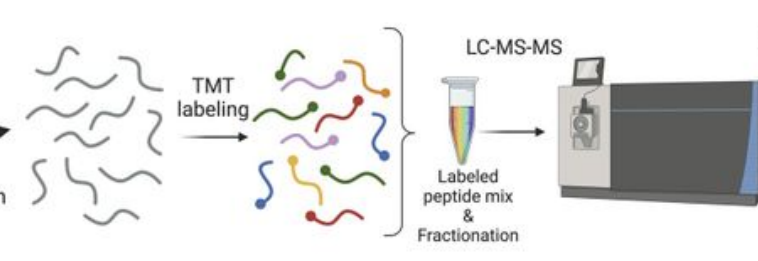

24hpi Mock vs Infected Mock vs Infected

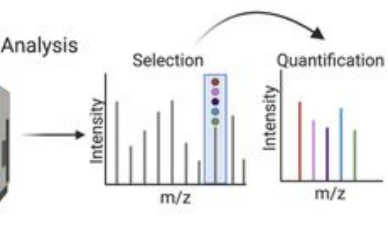

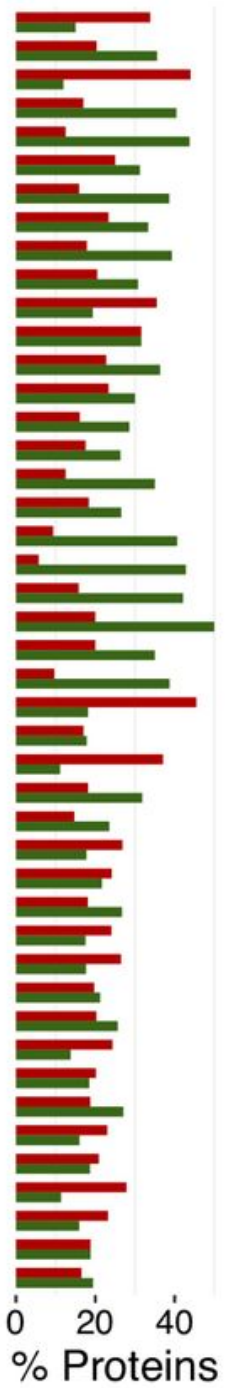
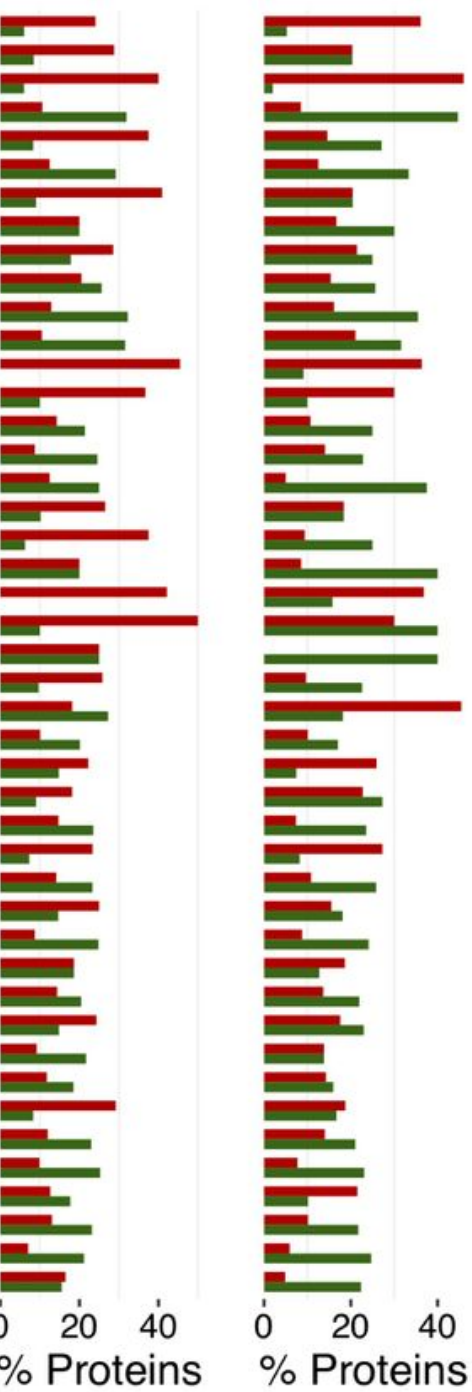

Adj.Pvalue

$1 \mathrm{e}-10 \bigcirc 1 \mathrm{e}-03$ $1 \mathrm{e}-04 \bigcirc 3 \mathrm{e}-02$

\section{\# Proteins}

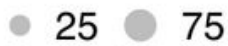

$50 \bigcirc 100$
Up

Down 
Figure 3

LC-MS/MS based proteomics analysis in CCHFV infected Huh7 cells. (A) General work-flow showing the sample preparation and data generation for the proteomics experiment. (B) Significant pathways identified by mapping proteins regulated over time to the KEGG database. The pathways are grouped into two: the first 29 pathways are metabolism related and the last 16 pathways are related to organismal systems and environmental information processing. The bubble size and color gradient correspond to the number of proteins and adjusted $P$ value of the enrichment test, respectively. The bar graphs represent the percentage of proteins upregulated or downregulated in each pathway as per the corresponding comparisons. Orange dot indicate metabolic process and blue dot indicate signaling pathway.

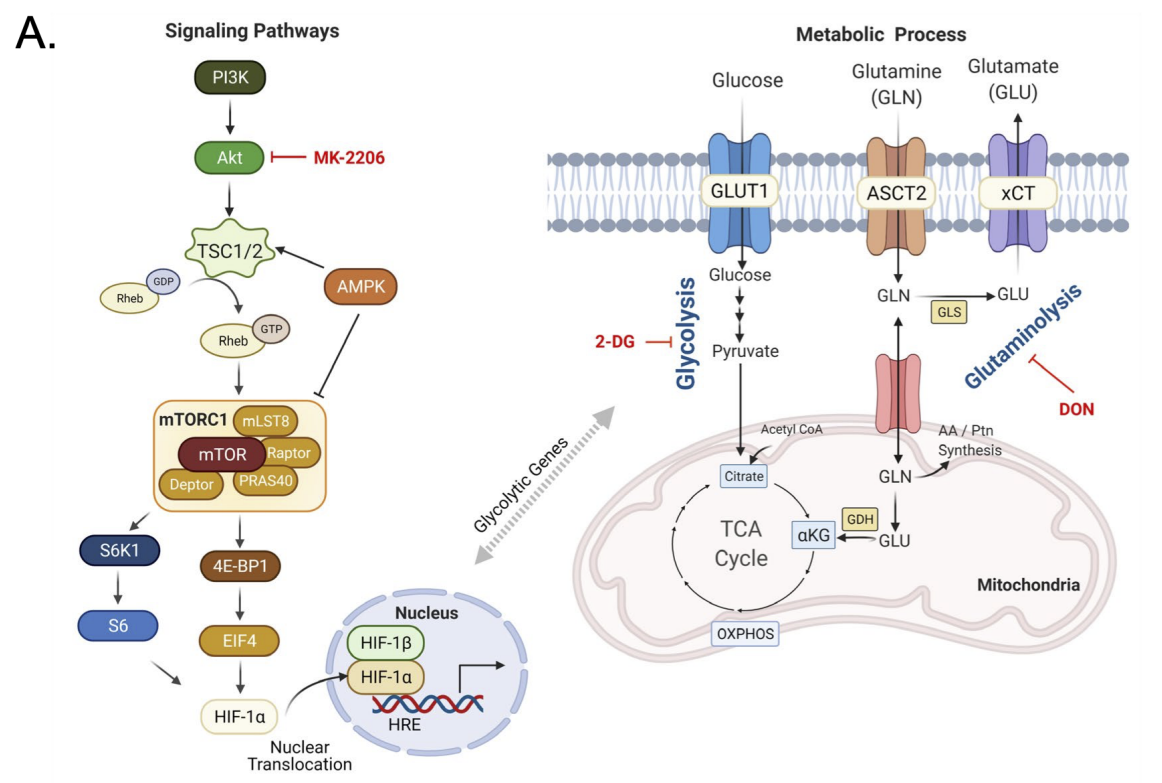

B.

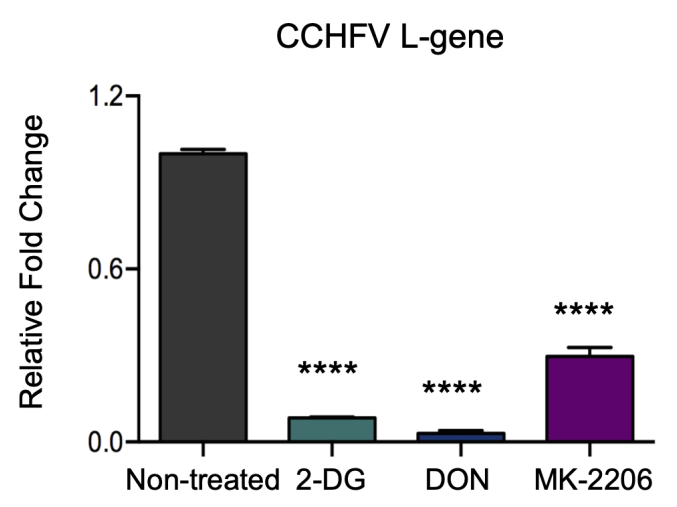

C.
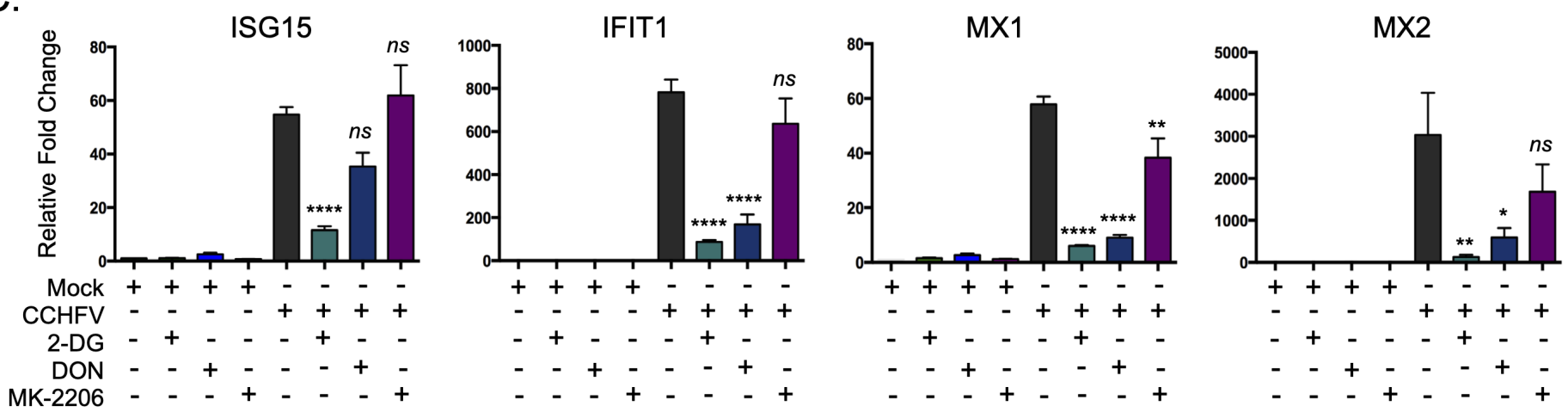

\section{Figure 4}

Metabolic control of viral replication in vitro. (A) Schematic representation of the PI3K/Akt/mTOR, glycolysis and glutaminolysis pathways and the drug targets used in the in vitro study. The Fig. is created with BioRender.com. (B) Fold change of the CCHFV L-gene following infection and treatment of 2-DG, DON and MK2206 compared to the untreated SW13 cells. ${ }^{\star \star \star \star} p<0.0001$. (C) Fold change of the human 
ISG15, IFIT1, MX1 and MX2 following infection and treatment of 2-DG, DON and MK2206 compared to the untreated SW13 cells. ${ }^{* * *} p<0.0001,{ }^{* *} p<0.001,{ }^{*} p<0.005,{ }^{*} p<0.05$ and ns non-significant.
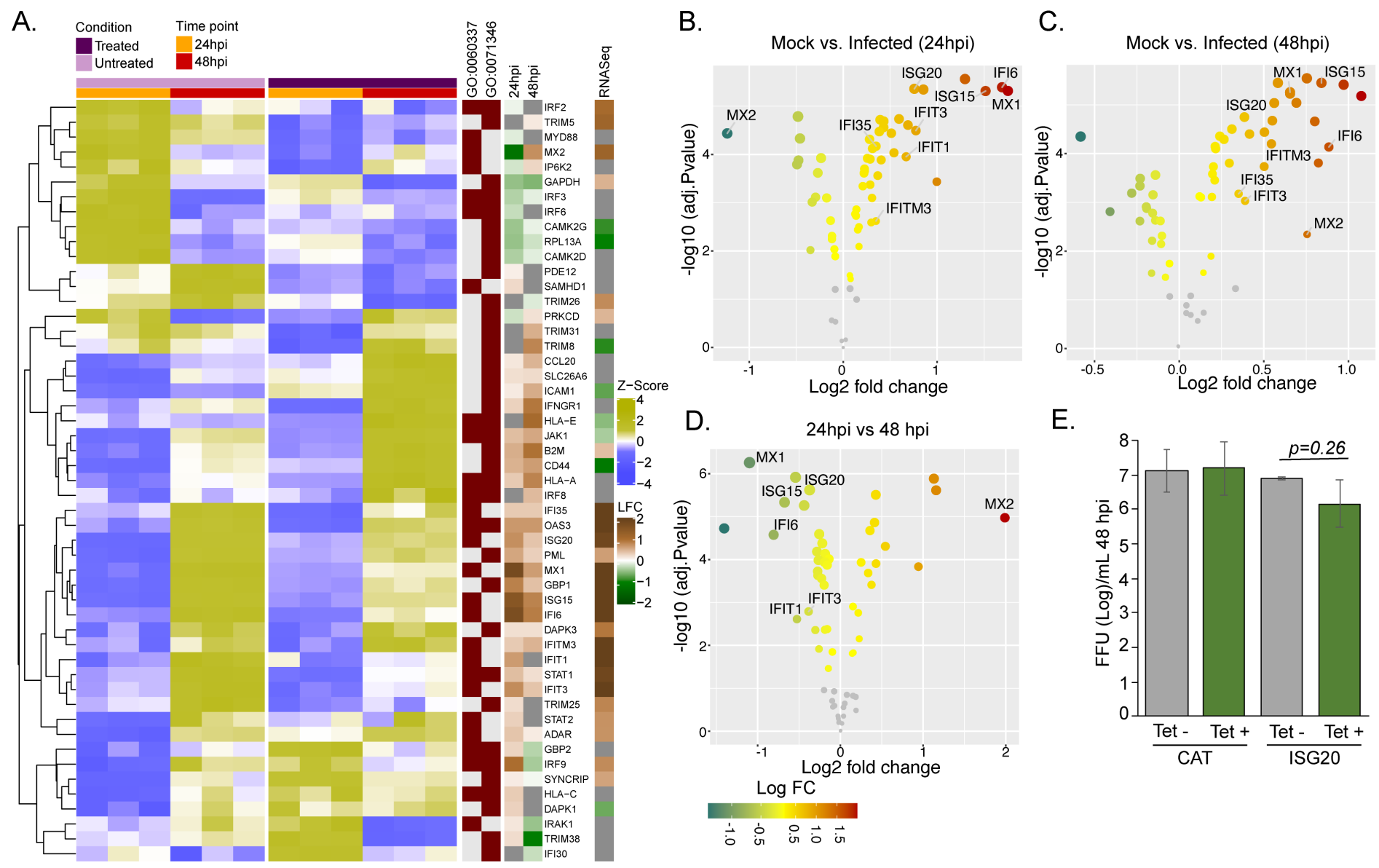

E.

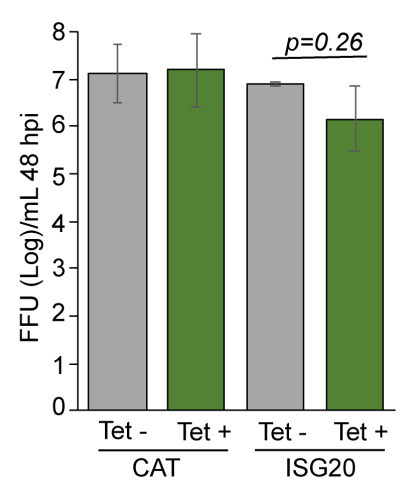

\section{Figure 5}

Temporal dynamics of interferon stimulating genes (ISGs). (A) Heatmap of Z-score transformed expression values of proteins belonging to the cellular response to IHN-y (G0:0071346) and the IFN-I interferon signaling pathway (G0:0060337) gene ontology terms. The columns represent the different sampls and their corresponding treatment conditions and time points. The rows represent proteins hierarchically clustered based on Euclidean distance. The row annotation indicates the presence or absence of proteins in the corresponding GO terms and log 2-fold change values in the proteomics and transcriptomics (recovered -vs- acute) experiments. (B) Volcano plot of ISGs visualizing the expression status of $24 \mathrm{~h}$ Mock and Infected (24 hpi) samples. The size and colour gradients of the dots correspond to the adjusted $P$ values of differential expression analysis and the log2 fold change, respectively. (C) Volcano plot of ISGs visualizing the expression status of $48 \mathrm{~h}$ Mock and Infected (48 hpi). The size and colour gradient of the dots correspond to the adjusted $\mathrm{P}$ values of differential expression analysis and $\log 2$ fold change. respectively. (D) Volcano plot of ISGs visualizing the expression status of 24 hpi and 48 hpi. The size and colour gradient of the dots correspond to the adjusted $P$ value of differential expression analysis and the log2 fold change, respectively. (E) HEK293-FLP-IN T-Rex cells that conditionally express ISG20 or human CAT protein were treated with doxycycline or left untreated for $36 \mathrm{~h}$, followed by infection with CCHFV at an MOI 1 for 48hours. Cell-free culture supernatants were harvested, and progeny viral 
titers were determined by fluorescent focus-forming assay. Columns show means \pm the standard deviations $(n=2)$. A two-tailed paired Student $t$ test was performed to compare titers of untreated and doxycycline-treated cells.

\section{Supplementary Files}

This is a list of supplementary files associated with this preprint. Click to download.

- SupplimentaryFigures.pdf

- SupplementaryData1.xlsx

- SupplementaryData2.xlsx 